\title{
Correction to: Infinite Dimensional Holomorphic Homogeneous Regular Domains
}

\section{Cho-Ho $\mathrm{Chu}^{1} \cdot \mathrm{Kang}-\mathrm{Tae} \mathrm{Kim}^{2} \cdot$ Sejun $\mathrm{Kim}^{2}$}

Published online: 5 March 2021

() Mathematica Josephina, Inc. 2021

\section{Correction to: The Journal of Geometric Analysis (2020) 30:223-247 https://doi.org/10.1007/s12220-019-00145-y}

There is a gap in the proof of the last assertion of Lemma 2.3 in the paper [2] which appeared in Volume 30 of this journal. The arguments given there are only valid in finite dimensions since the boundary $\partial \Omega$ need not be weakly closed in infinite dimension. In what follows, we present a complete proof including the infinite dimensional case and amend the statement of the last assertion in this lemma for a bounded convex domain $\Omega$.

Lemma 2.3 was only used in the proof of Theorem 5.3, which can be replaced by the amended lemma below without affecting the rest of the original proof of the theorem.

Amended Lemma 2.3 Let $\left(p_{k}\right)$ be a sequence in a bounded convex domain $\Omega$ in an isomorph $V$ of a Hilbert space such that $\lim _{k \rightarrow \infty} \sigma_{\Omega}\left(p_{k}\right)=0$. Then there is a subsequence $\left(p_{j}\right)$ of $\left(p_{k}\right)$ such that

$$
\lim _{j \rightarrow \infty} d\left(p_{j}, \partial \Omega\right)=0
$$

Further, there is a sequence $\left(p_{j}^{\prime}\right)$ in $\Omega$ such that $\lim _{j \rightarrow \infty} \sigma_{\Omega}\left(p_{j}^{\prime}\right)=0$, and for each $j$, there exists a boundary point $q_{j} \in \partial \Omega$ with $\left\|p_{j}^{\prime}-q_{j}\right\|=d\left(p_{j}^{\prime}, \partial \Omega\right)$.

The original article can be found online at https://doi.org/10.1007/s12220-019-00145-y.

$凶$ Cho-Ho Chu

c.chu@qmul.ac.uk

Kang-Tae Kim

kimkt@postech.ac.kr

Sejun Kim

rlatpwns21@postech.ac.kr

1 School of Mathematical Sciences, Queen Mary, University of London, London E1 4NS, UK

2 Center for Geometry and its applications and Department of Mathematics, Pohang University of Science and Technology, Pohang 790-784, South Korea 
Proof We only need to prove the last assertion. Let $\left(p_{j}\right)$ satisfy

$$
d\left(p_{j}, \partial \Omega\right) \rightarrow 0 \text { as } \quad j \rightarrow \infty
$$

We will make use of a result in [1, Theorem 3.2], which states that in a reflexive Banach space $V$, if the complement $V \backslash C$ of a non-empty closed set $C$ in $V$ is convex, then $C$ is almost proximinal, in other words, there is a dense $G_{\delta}$ set $A$ in $V \backslash C$ such that for each $x \in A$, there is a point $z \in C$ satisfying

$$
\|x-z\|=d(x, C)
$$

The given Banach space $V$ is reflexive. We apply the above result to the set $C=$ $V \backslash \Omega$, which is almost proximinal. By continuity of the squeezing function $\sigma_{\Omega}$, there is an open neighbourhood $N_{j}$ of $p_{j}$ such that $\sigma_{\Omega}(x)<2 \sigma_{\Omega}\left(p_{j}\right)$ for all $x \in N_{j}$ and for each $j$. By density of $A$, one can find $p_{j}^{\prime} \in A \cap N_{j}$ for which there exists $q_{j} \in C$ satisfying

$$
\left\|p_{j}^{\prime}-q_{j}\right\|=d\left(p_{j}^{\prime}, C\right)=d\left(p_{j}^{\prime}, V \backslash \Omega\right) \leq d\left(p_{j}^{\prime}, \partial \Omega\right)
$$

where the last inequality holds because the boundary $\partial \Omega$ is contained in $V \backslash \Omega$.

If $q_{j} \notin \partial \Omega$, then $q_{j} \notin \bar{\Omega}$ since $q_{j} \notin \Omega$. Since all points in the open convex set $\Omega$ are internal points [3, p. 413], the line segment $\left\{p_{j}^{\prime}+\alpha\left(q_{j}-p_{j}^{\prime}\right): 0 \leq \alpha \leq 1\right\}$ joining $p_{j}^{\prime}$ and $q_{j}$ must intersect the boundary $\partial \Omega$ at some point $\omega=p_{j}^{\prime}+\beta\left(q_{j}-p_{j}^{\prime}\right) \in \partial \Omega$ with $0<\beta<1$. It follows that

$$
\left\|p_{j}^{\prime}-q_{j}\right\| \leq d\left(p_{j}^{\prime}, \partial \Omega\right) \leq\left\|p_{j}^{\prime}-\omega\right\|=\beta\left\|p_{j}^{\prime}-q_{j}\right\|<\left\|p_{j}^{\prime}-q_{j}\right\|
$$

which is impossible. Hence we have $q_{j} \in \partial \Omega$ and $\left\|p_{j}^{\prime}-q_{j}\right\|=d\left(p_{j}^{\prime}, \partial \Omega\right)$.

Finally, $\sigma_{\Omega}\left(p_{j}^{\prime}\right)<2 \sigma_{\Omega}\left(p_{j}\right)$ for all $j$ implies $\lim _{j} \sigma_{\Omega}\left(p_{j}^{\prime}\right)=0$.

\section{References}

1. Borwein, J.M., Fitzpatrick, S.: Existence of nearest points in Banach spaces. Can. J. Math. 41, 702-720 (1989)

2. Chu, C-H., Kim, K-T., Kim, S.: Infinite dimensional holomorphic homogeneous regular domains, J. Geom. Anal. 30, 223-247 (2020) arXiv:1808.03416

3. Dunford, N., Schwartz, J.T.: Linear Operators. Wiley Classics Library Edition, New York (1988)

Publisher's Note Springer Nature remains neutral with regard to jurisdictional claims in published maps and institutional affiliations. 